\title{
La sexualité infantile: \\ Rabelais, Freud, et Jung
}

\author{
PIERRE GOUMARRE
}

En présentant l'enfance comme une période florissante pour la sexualité, Freud était persuadé qu'il faisait oeuvre originale, comme le montrent ces lignes dans les Trois Essais sur la Théorie de la Sexualité:

Il est généralement admis que la pulsion sexuelle fait défaut à l'enfance et ne s'éveille que dans la période de la puberté. C'est là une erreur lourde de conséquences. . On trouve, il est vrai, dans la littérature, quelques observations relatives à des actes de sexualité prématurée chez les petits enfants - érection, masturbations, et même simulacres de coït -, mais toujours cités comme cas exceptionnels, extraordinaires, des exemples repoussants de dépravation précoce. ${ }^{1}$

Mais ces lignes ne manquent pas de surprendre lorsque l'on sait que Rabelais n'ignorait rien de la sexualité infantile, et qu'il ne l'a nullement présentée comme quelque chose de repoussant, ni même comme quelque chose d'extraordinaire. Elles surprennent d'autant plus que Freud connaissait assez bien l'oeuvre de Rabelais pour la citer à six reprises. ${ }^{2}$ Notre propos consistera en tout cas à confronter les théories freudiennes et les aperçus rabelaisiens sur la vie sexuelle des enfants.

$$
\text { * * * }
$$

Freud n'a cessé d'insister sur l'importance de la sexualité infantile. En 1905, dans les Trois Essais, il faisait ainsi remarquer que "dès 1896," il avait noté "l'importance des premières années de la vie dans la production de certains phénomènes essentiels dépendant de la vie sexuelle." (p. 69) En 1916, dans l'Introduction à la Psychanalyse, il précisait qu'il "qualifi [ait] de sexuelles les activités douteuses et indéfinissables de la première enfance ayant le plaisir pour objectif." ${ }^{3}$ En 1925, dans une courte étude, il exprimait triomphalement sa conviction que "la psychanalyse a réglé son compte, une fois pour toutes, au conte de fée d'une enfance asexuée." "Elle a démontré le fait," ajoutait-t-il, "que les pré- 
occupations et les activités sexuelles se manifestent chez les petits enfants." 4

Pour Freud, l'enfant n'est pas seulement un être sexuel, il est aussi un "pervers polymorphe." 5 Cette expression surprendra, et elle choquera, mais on doit reconnaître à son auteur le mérite d'avoir essayé de l'expliquer et de la justifier. Voici ce qu'il écrit sur l'emploi du mot pervers:

Toutes les tendances perverses plongent par leurs racines dans l'enfance ..., les enfants portent en eux toutes les prédispositions à ces tendances qu'ils manifestent dans la mesure compatible avec leur immaturité, bref. . . la sexualité perverse n'est pas autre chose que la sexualité infantile grossie et décomposée en ses tendances particulières. ... Si l'enfant possède une vie sexuelle, celle-ci ne peut être que de nature perverse attendu que. . . il lui manque tout ce qui fait de la sexualité une fonction de procréation. Ce qui caractérise. . . toutes les perversions, c'est qu'elles méconnaissent le but essentiel de la sexualité, c'est-à-dire la procréation. (Introduction, pp. 290 et 296)

Quant au mot polymorphe, il se justifie par le fait que toutes les parties du corps sont "érogènes," i.e. susceptibles d'excitation sexuelle: "une zone érogène est une région de l'épiderme ou de la muqueuse qui, excitée de certaine façon, procure une sensation de plaisir d'une qualité particulière. ... N'importe quelle région de l'épiderme ou de la muqueuse peut servir de zone érogène." (Trois Essais, pp. 76-77) Dans la même oeuvre, l'auteur ajoute que, si "tout organe sensoriel, et probablement tout organe quelconque" peut faire fonction de zone érogène, "il existe certaines zones privilégiées dont l'excitabilité est assurée, dès le début, par certains dispositifs organiques." (p. 148) Ces zones privilégiées de la sexualité, ce sont non seulement les parties génitales, mais aussi - chez les enfants en particulier - l'anus, la bouche, la peau, et les yeux.

Dans l'évolution du petit pervers, le père de la psychanalyse distingue trois stades:

\begin{abstract}
Dans la vie sexuelle de l'enfance, existe, dès le début, un commencement d'organisation entre les composantes pulsionnelles sexuelles. Dans une première phase, qui se place très tôt, l'érotisme oral est prépondérant; une deuxième organisation "prégénitale" est caractérisée par la prédomineuse du sadisme et de l'érotisme anal; c'est seulement dans la troisième phase que la vie sexuelle est déterminée par la contribution qu'apportent les zones génitales proprement dites.
\end{abstract}

Dans ce passage, qui est un ajout de 1920 aux Trois Essais (p. 149), sont donc définis deux stades prégénitaux, à savoir le stade oral et le stade sadique-anal, et un stade à composante génitale, que l'auteur appellera plus tard (en 1923) le stade phallique. ${ }^{6}$

Dans les Trois Essais, l'auteur présente le stade oral en écrivant que 
"la succion," qui est le "type des manifestations sexuelles de l'enfance," "s'accompagne souvent d'attouchements répétés de la poitrine et des parties génitales externes." "Les enfants," ajoute-t-il, "passent souvent de la succion à la masturbation." (pp. 72 et 73) Il précise ensuite que, "si cette sensibilité érogène de la zone labiale persiste, l'enfant sera plus tard un amateur de baisers, recherchera les baisers pervers et, devenu homme, il sera disposé à être buveur et fumeur." (p. 75) Dans un ajout de 1915, il explicite sa pensée en écrivant que "le suçotement est un acte sexuel." (p. 73) Dans l'Introduction, il insiste sur la même idée en soulignant les mots importants: "Nous désignons la zone bucco-labiale comme une zone érogène et considérons le plaisir procuré par l'acte de sucer comme un plaisir sexuel." (p. 293)

L'auteur présente ensuite le stade anal en expliquant que la zone anale, dont la valeur érogène est "considérable," est, tout comme la zone buccolabiale, source d"“activité sexuelle." "Les enfants qui utilisent l'excitabilité érogène de la zone anale," précise-t-il, "se trahissent parce qu'ils retiennent leurs matières fécales, jusqu'à ce que l'accumulation de ces matières produise des contractions musculaires violentes et que, passant par le sphincter anal, elles provoquent sur la muqueuse une vive excitation." Aussi bien que le stade oral, ce stade est marqué par des activités auto-érotiques: "L'excitation masturbatoire de la zone anale à l'aide du doigt. . . n'est pas rare dans la deuxième enfance." (Trois Essais, pp. 79, 80 et 82 ) Et il est tout aussi riche de volupté: “L'élimination de l'urine et du contenu intestinal est pour le nourrisson une source de jouissance .... [Celui-ci] s'efforce bientôt d'organiser ces actions de façon qu'elles lui procurent le maximum de plaisir." (Introduction, p. 294)

Le stade anal est aussi le stade où culmine la cruauté de l'enfant, au point que Freud l'appelle le stade sadique-anal. L'auteur des Trois Essais souligne à plusieurs reprises cette coïncidense du sadisme et de l'érotisme anal (pp. 89, 93, 96), sans toutefois en donner une explication satisfaisante. Mais on doit à deux de ses disciples une explication qui est, sinon convaincante, du moins cohérente: "Le sadisme, par nature bipolaire - puisqu'il vise contradictoirement à détruire l'objet et à le maintenir en le maîtrisant - trouverait sa correspondance privilégiée dans le fonctionnement biphasique du sphincter anal (évacuationrétention) et le contrôle de celui-ci." "

Vient ensuite le stade phallique, qui se distingue des deux stades précédents sur deux points importants: les organes génitaux s'imposent de plus en plus au détriment des autres zones érogènes, et l'auto-érotisme tend à s'estomper au profit de l'amour objectal. Freud explique que cette phase est déjà très similaire à la dernière phase de la sexualité, la phase proprement génitale qui commence à la puberté. Mais, ajoute-t-il, "il existe une différence essentielle. . . cette phase ne connaît qu'une 
seule sorte d'organe génital, l'organe masculin. C'est pour cette raison que je l'ai nommée stade d'organisation phallique." (Trois Essais, p. 182) Au cours de cette phase, en effet, l'enfant - garçon ou fille - croit qu'il n'y a qu'un organe génital, c'est-à-dire l'organe mâle. ${ }^{8}$ Incapable d'admettre, comme l'adulte, l'existence d'une sexualité féminine, il considère que l'alternative consiste à avoir un penis, ou à être châtré.

Le stade phallique est suivi non par un autre stade d'évolution, mais par "un temps d'arrêt ou de régression": "Après une courte période d'épanouissement, qui va de la deuxième à la cinquième année," l'activité sexuelle de l'enfant entre dans une période de latence. Cette période, qui dure quelques années, se termine avec la puberté. De nouvelles transformations se produisent alors, "qui amèneront la vie sexuelle infantile à sa forme définitive et normale." (Trois Essais, p. 111) La sexualité sera désormais caractérisée par le primat des organes génitaux.

Il faut noter que l'auteur n'attribue pas une durée précise à chaque phase. Les quelques indications chiffrées qu'il fournit sont pour la plupart accompagnées de l'adverbe "environ," et du reste elles varient sensiblement d'une oeuvre à l'autre. Ces indications permettent toutefois de penser que, selon lui, les stades oral et sadique-anal couvrent les trois premières années, le stade phallique s'étendant sur les troisième, quatrième et cinquième années. Quant à la période de latence, elle commence à la cinquième ou sixième année, et se termine entre huit et douze ans, selon les individus. ${ }^{10}$

"A boyre! à boyre! à boyre!" Dès sa naissance, Gargantua entre à grands cris dans sa phase orale. "Au seul son des pinthes et flaccons," précise Rabelais, "il entrait en ecstase comme s'il goustoit les joyes du paradis." 11 Du point de vue freudien, il ne fait aucun doute que cette extase est de nature sexuelle. Voici en effet ce qu'écrit l'auteur des Trois Essais:

Quand on a vu l'enfant rassasié abondonner le sein, retomber dans les bras de sa mère, et les joues rouges, avec un sourire heureux, s'endormir, on ne peut manquer de dire que cette image reste le modèle et l'expression de la satisfaction sexuelle qu'il connaîtra plus tard. (p. 75)

Mais les plaisirs de la bouche en entraînent d'autres: non content de "se conchi [er] à toutes heures," Gargantua s'amusait à "baryton [er] du cul." (p. 34) Brûlant les étapes, le jeune géant est déjà en pleine phase anale.

Comme son père, le jeune Pantagruel se signale tout de suite par une grande sensibilité orale: "A chascun de ses repas il humoit le laict de 
quatre mille six cens vaches." (p. 235) Mais il se distingue de lui par son agressivité et sa cruauté. C'est ainsi qu'un jour, saisissant la vache qu'il têtait "par dessoubz le jarret," il "luy mangea les deux tetins et la moytié du ventre, avecques le foye et les roignons, et l'eust toute devorée, n'eust été qu'elle cryoit horriblement." Un autre jour "il rompit un grand morceau" de son auge avec ses dents. Une autre fois "il rompit le bout de son berceau" avec ses pieds pour pouvoir participer à un banquet. Et il lui arriva même de s'en prendre à un ours, qu'il "mist en pieces comme un poulet" et dont il "fist une bonne gorge chaulde." Présentant la cruauté comme un "facteur de la composante sexuelle," Freud explique que "l'enfant est, en général, porté à la cruauté, car la pulsion de maîtriser n'est pas encore arrêtée par la vue de la douleur d'autrui, la pitié ne se développant que relativement tard." (Trois Essais, p. 89)

Mais revenons à Gargantua, qui entre maintenant dans sa deuxième enfance. Ses activités orales sont aussi importantes que dans le passé: "Depuys les troys jusques à cinq ans," il passait son temps "comme les petits enfants dupays: c'est assavoir à boyre, manger et dormir; à manger, dormir et boyre; à dormir, boyre et manger." (p. 48) Et ses activités anales (au sens freudien de ce terme) sont toujours aussi riches: "Il pissoit sur ses souliers, il chyoit en sa chemise. . pettoyt de gresse, pissoit contre le soleil. . .mangeoyt choux et chyoit pourrée." L'enfant, commente l'auteur de l'Introduction, "semble être particulièrement fier des exploits qu'il rattache à l'acte d'uriner." (p. 295) Parmi les nombreuses autres activités du jeune géant, nous nous devons de citer celles qui, dans une optique freudienne, sont manifestement auto-érotiques: "[II] se frottoit ordinairement le ventre d'un panier. . se chatouilloyt pour se faire rire. . se grattoyt où ne lui demangeoyt point." Il est vrai qu'il n'hésitait pas, à l'occasion, à partager ses jeux et ses plaisirs avec les petits chiens de son père: "Il leurs mordoit les aureilles; ilz luy graphinoient le nez, il leurs souffloit au cul, ilz luy lechoient les badigoinces."

Ce qui est nouveau, entre trois et cinq ans, c'est-à-dire à l'époque du stade phallique, c'est selon Freud l'éclosion de la sexualité génitale et objectale. Gargantua n'est pas en retard: "Ce petit paillard toujours tastonnoit ses gouvernantes, cen dessus dessoubz, cen devant derrière harry bourriquet! - et desjà commençoyt exercer sa braguette." D'après l'auteur des Trois essais, les adultes qui s'occupent de l'enfant jouent un grand rôle dans l'éveil de sa sexualité:

Les rapports de l'enfant avec les personnes qui le soignent sont pour lui une source continue d'excitations et de satisfactions sexuelles partant des zones érogènes. Et cela d'autant plus que la personne chargée des soins (généralement la mère) témoigne à l'enfant des sentiments dérivant de sa propre vie sexuelle, l'embrasse, le berce, le considère, sans aucun doute, comme le substitut d'un objet sexuel complet. (p. 133) 
Les gouvernantes de Gargantua jouent ce rôle avec un plaisir manifeste: "un chascun jour," elles ornoient sa braguette "de beaulx boucquets de beaulx rubans, de belles fleurs," et "passoient leur temps à la faire revenir entre leurs mains. .. puis s'esclaffoient de rire quand elle levoit les aureilles." Cette dernière précision est tout à fait conforme à ce qu'écrira l'auteur de l'Introduction: "Dès la troisième année, la vie sexuelle de l'enfant ne présente plus le moindre doute. Dès cet âge, les organes génitaux deviennent susceptibles d'érection." (p. 306)

L'auteur des Trois Essais explique encore que, si "les enfants adorent certains jeux, tels que la balançoire" et "les secousses rythmiques" en général, c'est parce que ces jeux leur procurent un plaisir sexuel. On n'est donc pas étonné de lire que "affin que toute sa vie [Gargantua] feust bon chevaulcheur, l'on luy feist un beau grand cheval de boys, lequel il faissoit penader, saulter, voltiger, ruer et dancer tout ensemble." (p. 50) Si l'on songe à l'autre sens que Rabelais aime donner à l'adjectif "chevaucheur," on peut penser qu'en offrant un cheval de bois à son fils, Grandgousier voulait non seulement lui permettre de se distraire, mais aussi l'initier à la sexualité.

Le cadeau fut en tout cas bien reçu puisque Gargantua s'empressa de faire lui-même des chevaux et des mules, au point qu'il en eut finalement "dix ou douze à relays et sept pour la poste." Comme s'il commentait le texte de Rabelais, Freud écrit, "L'enfant s'attache aux problèmes sexuels avec une intensité imprévue et l'on peut même dire que ce sont là les problèmes éveillant son intelligence." (p. 91) L'intensité avec laquelle Gargantua s'intéressait à ses animaux se révèle dans le fait que, non content de s'en amuser le jour, il les "mettoit tous coucher auprès de soy" quand il se mettait au lit. Si l'on était surpris que le jeune géant puisse partager ses nuits avec des mules et des chevaux, on pourrait toujours se rappeler que, selon la théorie freudienne de la bisexualité, ${ }^{12}$ l'enfant distingue mal les frontières qui séparent l'hétérosexualité de l'homosexualité.

Mises en éveil par le jeu, les facultés créatrices de Gargantua peuvent maintenant s'appliquer à une chose qui n'est peut-être pas plus sérieuse, mais qui est en tout cas plus utilitaire: "Sus la fin de la quinte année," raconte l'auteur, il a, "par longue et curieuse experience," "inventé un moyen de torcher le cul le plus seigneurial, le plus excellent, le plus expedient que jamais feut veu." Comme le remarque pertinemment Michel Beaujour, "désormais tout est changé. Il ne s'agit plus d'inventer des jouets, tels des chevaux de bois, mais un objet utile par un processus de sélection quasi-scientifique." ${ }^{13}$ Et l'expérience est aussi agréable qu'elle est utile: "En tout," déclare le jeune inventeur, "je trouvay de plaisir plus que ne ont les [galeux] quand on les estrille." "Vous sentez au trou du cul," ajoute-t-il, "une volupté mirificque." 
Mais l'on sait depuis Freud qu'un jeune garçon peut être attiré libidineusement par une fille. Depuis Freud, ou plutôt depuis Rabelais, comme l'atteste le rondeau composé par le jeune géant:

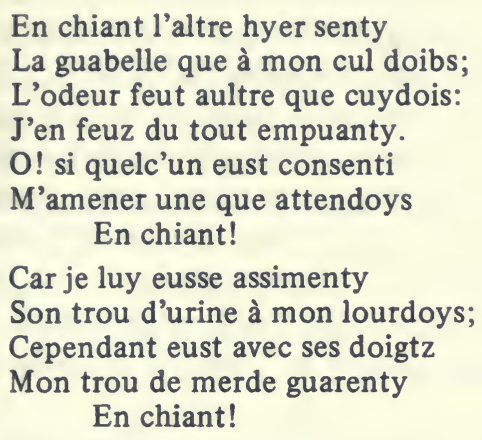

En parlant d'un "trou d'urine," Gargantua révèle qu'il continue à avoir le langage d'un petit garçon, mais qu'il commence à voir les goûts d'une grande personne. C'est d'autant plus remarquable qu'il en est à la fin de sa cinquième année. Pour Freud, cet âge est celui où finit le stade phallique, et où débute la période de latence. Chez Rabelais, si l'on se fie à ce rondeau, c'est l'âge où l'enfant liquide sa phase phallique, et entre résolument dans la phase génitale. Brûlant encore une fois les étapes, Gargantua est déjà sur les chemins de la puberté, ce stade où, selon l'expression de Freud, "monte la grande marée des besoins sexuels." 14

Il n'est donc pas difficile de faire une lecture freudienne du texte rabelaisien. L'auteur de Gargantua et l'auteur des Trois Essais se rejoignent souvent dans leurs points de vue sur l'enfance. L'un et l'autre présentent l'enfant comme un être préoccupé avant tout par les fonctions corporelles. Ni l'un ni l'autre ne le présentent comme un être pur qui évolue dans un vert paradis d'innocentes amours.

$$
\text { * * * }
$$

En concluerons-nous que Rabelais peut être considéré comme un précurseur de Freud? Non! Faire de Rabelais un précurseur, ce serait suggérer qu'il a eu le mérite d'entrevoir une vérité que Freud aurait eu plus tard la gloire d'énoncer clairement et explicitement. Or il nous apparaît que Rabelais est en la matière beaucoup plus qu'un précurseur, et que si son point de vue est intéressant, c'est surtout dans la mesure où il est différent de celui de Freud. Du reste, comme nous allons le voir, son point de vue est souvent confirmé par les disciples et critiques du père de la psychanalyse.

Les observations de Rabelais sur la vie infantile s'opposent à celles de Freud sur quelques points, et d'abord sur la question de la succession 
des phases. Alors que, selon Freud, la phase orale précède la phase anale, chez Rabelais ces phases sont concomitantes. Imputerons-nous cette absence de distinction à une faute d'observation? Ce serait d'abord oublier que Freud lui-même n'était pas sûr d'avoir eu raison de présenter ces deux phases comme successives, puisqu'il lui est arrivé d'écrire que "la phase orale est peut-être la plus primitive." (Introduction, p. 309 C'est nous qui soulignons. $)^{15}$ Ce serait encore oublier qu'un Jung n'établit aucune séparation temporelle entre ces phases, et estime simplement que "les premières années de la vie" "sont caractérisées presque exclusivement par les fonctions de nutrition et de croissance." ${ }^{16}$ Ce serait enfin oublier qu'aujourd'hui, parmi les psychologues de l'enfant, seuls ceux qui sont d'obédience freudienne sont d'accord pour distinguer deux stades au cours desquels prédomineraient successivement la sensibilité orale et la sensibilité anale.

Un autre point de divergence porte sur ce que Freud appelle le sadisme de l'enfant. Le jeune Pantagruel fait preuve, nous l'avons vu, de beaucoup de cruauté, en particulier à l'encontre des animaux. Mais il s'agit d'une cruauté qui - dans ses moyens et dans ses buts - est essentiellement orale, alors que, d'après Freud, le sadisme caractérise surtout le stade anal. Reprocherons-nous dès lors à Rabelais d'avoir mal compris le "sadisme" de l'enfant? Ce serait méconnaître le point de vue d'une Mélanie Klein, selon laquelle "le stade oral est le moment culminant du sadisme infantile." Ce serait également méconnaître l'opinion d'un Karl Abraham, lequel a introduit l'expression "stade sadique-oral", un stade selon lui "marqué par l'apparition des dents et de l'activité de morsure."17 Ces deux psychanalystes distinguent, comme Freud, une période orale et une période anale mais, comme Rabelais, ils conçoivent l'agressivité infantile comme un phénomène qui est encore plus oral qu'anal.

On se rappelle d'autre part qu'à la fin de la cinquième année, Gargantua, loin d'entrer dans une période d'accalmie sexuelle, est au contraire assailli par de nouveaux désirs sexuels. Pour la première fois, sa libido est excitée par l'organe sexuel féminin, et c'est dire qu'il est en train de sortir de ce que Freud appelle le stade phallique. C'est dire aussi que dans l'esprit de Rabelais, il n'y a pas de période de latence. Faut-il lui en faire grief? Nous préférons remarquer que l'existence de cette période est contestée par plusieurs disciples de Freud. Wilhelm Reich, par exemple, affirme qu' "il n'y a pas de période de latence quand la sexualité se développe d'une façon naturelle." L'auteur des Trois Essais, explique-t-il, prétend que la période de latence est "de nature biologique" alors qu'elle est "un produit contre nature de la civilisation." ${ }^{18}$ Quant à Jung, il va encore plus loin, puisqu'il voit dans la période de latence "un exemple frappant de l'inexactitude" du point de vue freudien sur la sexualité infantile. Ce que Freud appelle la latence de la sexualité, écrit-il, dans la Théorie 
psychanalytique, est au contraire - et c'est lui qui souligne - "le vrai début de la sexualité", ce qui précède n'ayant pas un "véritable caractère sexuel." (p. 79)

Dans son observation de la vie infantile, Rabelais est donc en désaccord avec Freud sur trois points, mais sur trois points qui sont contestables, et qui ont été contestés par d'autres théoriciens de la psychanalyse. Dans l'interprétation des faits observés, nous allons le voir, les divergences sont encore plus profondes, et elles portent également sur les aspects les plus discutables de la pensée freudienne.

Une première différence d'interprétation porte sur la nature de la sexualité infantile. Freud pense qu'il y a deux sexualités - la "normale," qui a pour objet essentiel la procréation, et la "perverse", qui vise exclusivement au plaisir - et il en conclut logiquement que la sexualité des enfants est perverse. Une telle conception est évidemment tout à fait étrangère à l'esprit de Rabelais. Un adulte comme Panurge est très porté sur "la chose" et un enfant comme Gargantua s'y intéresse beaucoup, mais, même si l'un et l'autre peuvent être considérés comme des "cochons," ni l'un ni l'autre ne sont présentés comme des "pervers." ${ }^{9}$ Rabelais ne conçoit pas qu'il y ait une norme en matière de sexualité, et donc ne conçoit pas qu'il ait une sexualité perverse. La recherche du plaisir dans les activités sexuelles n'est pour lui ni normale ni perverse, mais seulement naturelle.

Mais est-il légitime de distinguer deux sexualités, et de qualifier de perverse toute sexualité qui a pour fin essentielle le plaisir? N'a-t-on pas le droit de voir, dans cette conception freudienne, un relent de moralisme bourgeois? Quoi qu'il en soit, on constate que Rabelais n'est pas le seul à avoir une conception différente. Reich, entre autres, reproche à Freud d'avoir mis la sexualité "au service de la procréation." "Ce n'est pas vrai," affirme-t-il, et "Freud le savait d'une certaine façon." Cet auteur, qui, dans l'esprit de Rabelais, conçoit la sexualité comme étant avant tout une source de plaisir, va jusqu'à écrire que, si Freud n'a pas fait sienne cette conception, c'est qu'il avait peur de choquer "le monde." 20

L'idée même de perversion a été l'objet de nombreuses critiques. Jacques Maritain, par exemple, affirme que l'expression "pervers polymorphe" est "absurde," et qu'elle prouve que Freud "se complaît dans les généralisations les plus arbitraires." 21 Jung, de son côté, n'a pas manqué une occasion d'exprimer son opposition à cette idée. En 1915, dans la préface au Développement de la Personnalité, il écrivait, "Je conteste l'idée freudienne selon laquelle l'enfant a une disposition perverse polymorphe." En 1938, dans la préface à la nouvelle édition de ce livre, il exprimait son regret que certains puissent encore accepter "le mythe de la sexualité polymorphe de l'enfant." 22 Dans la Théorie psychanalytique, il explique que les perversions sont "des troubles de la 
sexualité adulte," et que "les rudiments de la sexualité infantile," loin d'être des manifestations perverses, sont des stades normaux d'évolution qui préparent la sexualité adulte (pp. 43, 47, 78).

Une autre différence d'interprétation porte sur l'étendue de la sexualité infantile. Freud élargit le champ de la sexualité au point qu'il attribue à toutes les activités de l'enfant - aux activités orales et anales en particulier - un caractère sexuel. ${ }^{23}$ Rabelais, lui, n'a jamais été tenté par un tel élargissement. Dans son oeuvre, la digestion et la sexualité apparaissent toujours comme des fonctions bien distinctes. Pour lui, le plaisir de "se remplir la trippe" est une chose, le plaisir de "se torcher le cul" en est une autre, et autre chose encore est le plaisir de "se frotter le lard."

Dirons-nous que Rabelais n'a pas aperçu la dimension sexuelle des plaisirs oral et anal, et qu'en cela il a manqué de perspicacité? Nous préférons nous demander si la sexualisation de ces plaisirs est fondée. Il est vrai que Freud a essayé de la justifier. Voici ce qu'il écrit dans l'Introduction:

On nous dira: "pourquoi vous entêtez-vous à dénommer sexualité ces manifestations de l'enfance que vous considérez vous-même comme indéfinissables. ..? Pourquoi. . .ne diriez-vous pas tout simplement qu'on observe chez le nourrisson des activités qui, telles que l'acte de sucer et la rétention des excréments, montrent seulement que l'enfant recherche le plaisir qu'il peut éprouver par l'intermédiaire de certains organes? (p. 303)

En guise de réponse à cette objection, l'auteur affirme qu'il "a été conduit à cette manière de voir par des matériaux de nature incontestablement sexuelle. . . fournis [par] l'analyse des symptômes." Son raisonnement peut se résumer dans le syllogisme suivant: les adultes pervers trouvent leur plaisir sexuel dans des activités orales et anales; or les enfants trouvent leur plaisir dans des activités similaires; donc leur plaisir est également sexuel.

Mais comment ne pas voir que ce raisonnement repose sur un postulat? En quoi la nature sexuelle des plaisirs oral et anal chez les adultes nous obligerait-elle à conclure à la nature sexuelle de ces plaisirs chez les enfants? Les auteurs du Vocabulaire de la Psychanalyse - qui, dans leur lecture de Freud, ne pèchent pas, en général, par un excès d'esprit critique - font justement remarquer que la réflexion bute ici sur une "aporie," "Freud se contentant de postuler que la sexualité existe virtuellement d'emblée." (p. 455) Il faut ajouter que Freud lui-même doutait de la validité de sa démonstration, comme le suggère la conclusion inattendue qu'il lui donne: 'Quant à savoir si tous les plaisirs procurés par les organes doivent être qualifiés de sexuels. .. , c'est là une question que je ne puis 
discuter ici. Je sais peu de choses sur le plaisir procuré par les organes et sur ses conditions." (p. 305) Le père de la psychanalyse, précisons-le, ne reviendra jamais sur cette question.

L'extension que Freud donne au domaine sexuel sera naturellement considérée comme abusive par de nombreux commentateurs. Un auteur remarque ainsi qu'on trouve dans son oeuvre une sexualité "observée" et une sexualité "présumée," et il attribue la seconde à son "dogmatisme." 24 Un autre auteur écrit qu'en étendant considérablement le champ de la sexualité, Freud a fait "une réforme linguistique qu'on a eu tort d'interpréter comme une découverte." ${ }^{25} \mathrm{Et}$, d'une façon encore plus incisive, Jung souligne que "le concept freudien de sexualité est complètement élastique, et si vague qu'on peut y inclure presque tout." 26 Il revient ailleurs à la charge pour montrer que Freud a eu tort d'interpréter tous les plaisirs comme des plaisirs sexuels. "Si nous devions considérer la recherche du plaisir comme quelque chose de sexuel," ironise-t-il, nous devrions dire que "la faim est une pulsion sexuelle." Comme pour faire savoir qu'il rejette la conception freudienne de la sexualité infantile dans sa totalité, il précise, "Il est évident que l'acte de sucer ne peut être qualifié de sexuel.'"

En bref, ce que Jung reproche à son ancien mentor, c'est d'avoir fait de la sexualité un fourre-tout, et en particulier d'avoir sexualisé tous les aspects de la vie infantile. L'auteur de Gargantua - faut-il le répéter? ne mérite pas ce reproche. A la sexualité en général, et à celle des enfants en particulier, il accorde, comme Jung, une grande place, mais pas la place envahissante que lui accorde Freud. Pour lui, l'homme ne vit pas que de sexe, et l'enfant ne s'amuse pas que de touche-pipi, ni ne rêve que de "trou d'urine."

Cette similarité de vue entre Rabelais et Jung n'est du reste pas étonnante si l'on songe à ce que ce dernier écrit dans une oeuvre autobiographique: "J'ai grandi dans la campagne, parmi les paysans, et ce que je n'ai pas pu apprendre dans les étables, je l'ai puisé dans l'esprit rabelaisien."28 Il est en tout cas remarquable que, formé à l'école de Freud et inspiré par la pensée de Rabelais, le théoricien de l'inconscient collectif a sur la vie infantile un point de vue qui pour l'essentiel s'oppose à celui du Viennois, et dans l'ensemble confirme celui du Chinonais.

Nous permettra-t-on d'ajouter qu'à notre avis les courts aperçus de Rabelais sur la sexualité des enfants sont plus conformes à la réalité que les longues théories de Freud? Nous savons combien une telle opinion peut choquer à une époque où le freudisme est traité par le grand public comme une Vérité admise qui ne se discute pas, et par une certaine intelligentzia comme une Pensée sacrée qui ne saurait être mise en question. ${ }^{29}$ 
Freud dixit. Mais, nous rappelant que Rabelais lui-même ne considérait aucune vérité comme établie et irréfutable, nous pensons qu'il est légitime de faire de l'oeuvre freudienne une lecture rabelaisienne, c'est-àdire une lecture critique et libre de tout principe d'autorité, et par là même qu'il est permis de conclure que la réalité de la sexualité infantile se trouve davantage dans le Gargantua que dans les Trois Essais.

Nos lecteurs apprécieront du reste le mérite de Rabelais à sa juste valeur s'ils considèrent qu'il n'a jamais eu l'intention d'écrire un traité de psychologie, ${ }^{30}$ ni même une oeuvre réaliste. ${ }^{31} \mathrm{Ce}$ mérite, ils pourront en tout cas le discuter, pourvu qu'ils aient des enfants, ou que du moins ils se souviennent d'avoir été jeunes. ${ }^{32}$

\section{University of Western Ontario}

\section{Notes}

1 Sigmund Freud, Trois Essais sur la théorie de la sexualité (Paris: Gallimard, 1966), pp. 65-66.

2 Voir The Standard Edition of the Complete Psychological Works of Sigmund Freud (London: Hogarth), Vol. IV, p. 215; Vol. V, p. 469; Vol. 12, p. 200; Vol. 21, p.90. Voir aussi Collected Papers (New York: Basic), Vol. V, p. 106.

3 Sigmund Freud, Introduction à la psychanalyse (Paris: Payot, 1962), p. 305.

4 Collected Papers, Vol. V, p. 171.

5 Pour cette expression, voir en particulier: Trois Essais (op. cit.). pp. 86 et 1511 et The Standard Edition, Vol. XV, p. 209.

6 The Standard Edition, Vol. XIX, pp. 141-145.

7 J. Laplanche et J.B. Pontalis, Vocabulaire de la psychanalyse (Paris: P.U.F., 1967), p. 461.

8 The Standard Edition, Vol. XIX, pp. 144-145. Ce point de vue, d'après lequel la fille a la même attitude vis-à-vis de la sexualité que le garçon, a été l'objet de nombreuses critiques. Reprochant à Freud son "anti-féminisme," plusieurs auteurs font remarquer que la fille n'est pour lui qu'un "garçon mutilé" ou un "garçon châtré," Voir:

- Hélène Nahas, La femme dans la littérature existentielle (Paris: P.U.F., 1957), p. 28.

- Karen Horney, New Ways in Psychoanalysis (New York: Norton, 1939), pp. 101-110.

9 Sur cette question, voir Trois Essais, p. 147, et Introduction, p. 306.

10 Daniel Lagache, un disciple de Freud, assigne des limites précises aux divers stades. In La psychanalyse (Que sais-je? P.U.F., 1955), pp. 29-31.

Norbert Sillamy, un psychologue très favorable aux conceptions freudiennes, assigne à ces stades des limites également précises, mais qui ne coïncident pas exactement avec les précédentes. In Dictionnaire de la psychologie (Paris: Larousse, 1965), p. 284.

Didier Julia, un philosophe qui n'est pas particulièrement influencé par la psychanalyse, attribue aux stades freudiens des durées qui ne correspondent pas précisément à celles qu'on trouve chez Lagache ou chez Sillamy. In Dictionnaire de la philosophie (Paris: Larousse, 1964), p. 108.

André Hesnard, qui est considéré comme l'introducteur de la psychanalyse en France, donne des indications chiffrées très précises qui ne se trouvent pas toutes chez Freud. In La sexologie (Paris: Payot, 1959), p. 165.

11 François Rabelais, Oeuvres complètes (Paris: Garnier, 1962), Tome I, pp. 33-34. Toutes les citations utilisées dans cet article proviennent de l'édition Garnier. 
12 Sur cette notion, voir Vocabulaire de la Psychanalyse (op. cit.), p. 49.

13 Michel Beaujour, Le jeu de Rabelais (L'Herne, 1969), p. 74.

14 Sigmund Freud, Cinq leçons sur la psychanalyse (Paris: Payot, 1953), p. 164.

15 Ailleurs, Freud écrit que "les trois phases du développement de l'enfant ne se succèdent pas de façon rigoureuse." (Standard Edition, Vol. XIII, p. 155). Il n'empêche que, partout dans son oeuvre, il présente les trois phases comme successives.

16 C.G. Jung, The Theory of Psychoanalysis (New York: Johnson Reprint, 1970), p. 33.

17 A propos du sadisme oral, on lira, en plus des renseignements fournis dans le Vocabulaire de la Psychanalyse (p. 462):

- Mélanie Klein, New Directions in Psycho-analysis (New York: Basic, 1957), pp. 16-18.

- Karl Abraham, The Influence of Oral Erotism on Character-formation, in Selected Papers (New York: Basic, 1960), pp. 393-406.

18 Wilhelm Reich, The Function of the Orgasm (New York: Farrar, 1973), p. 231.

19 Rabelais n'exprime aucune désapprobation à l'encontre des activités sexuelles de Panurge, lesquelles ont uniquement le plaisir pour objectif. Mais nous ne voulons pas dire qu'il approuve tout ce qu'il fait. Entre l'auteur et son personnage, les relations sont ici très complexes. Sur cette question, voir en particulier Mary Ragland, Rabelais and Panurge (Amsterdam: Rodopi, 1976).

20 Reich Speaks of Freud, Edited by Mary Higgins and Chester M. Raphael (New York: Farrar, 1967), p. 19.

21 Jacques Maritain, "Freudism and Psychoanalysis," in Freud and the 20th Century (Cleveland: Meridian, 1957), p. 248.

22 C.G. Jung, The Development of Personality, in Collected Works (London: Routledge, 1954), Vol. XVII, pp. 5-7.

23 Nous ne pouvons pas aborder ici la question du "pansexualisme" de Freud. On connaît la polémique qui a opposé Jung et Freud, le premier accusant le second de mettre le sexe à toutes les sauces. Qu'il nous suffise d'indiquer ici les passages dans lesquels Freud se défend, ou attaque ses adversaires (Jung et Adler en particulier): Collected Papers: Vol. V, pp. 127 et 169. Standard Edition: Vol. XIV, p. 62; Vol. XVIII, p. 248; Vol. XX, p. 53. Voir aussi Trois essais, p. 12. On aura également intérêt à lire:

- Lilian Frey-Rohn, From Freud to Jung, (New York: G.P. Putnams Sons, 1974), pp. 135-157.

- Michel Foucault, La Volonté de Savoir, in Histoire de la Sexualité (Paris, Gallimard, 1976), pp. 209 à 211.

24 Peter Madison, Freud's Concept of Repression and Defense (Minneapolis: University of Minnesota Press, 1961), pp. 190-191.

25 Anthony Quinton, Freud, the Man, His World, His Influence, Edited by Jonathan Miller (Boston: Little and Brown, 1972), p. 81.

26 C.G. Jung. Modern Man in Search of a Soul (New York: Harcourt, 1960), p. 25.

27 Theory of Psychoanalysis (op. cit.), pp. 22-23. Un autre reproche qu'on a fait à Freud, c'est d'avoir confondu sexualité et affectivité, et en particulier de n'avoir pas vu que les liens qui unissent l'enfant à sa mère, loin d'être exclusivement sexuels, sont aussi une source de tendresse, de sécurité, et de joie. Voir à ce propos:

- Erich Fromm, The Crisis of Psychoanalysis (New York: Harcourt), pp. 73-74.

- Sophie Lazarsfeld, Essays in Individual Psychology (New York: Grove, 1959), p. 124.

28 C.G. Jung, Memories, Dreams, Reflections (New York: Pantheon, 1973), p. 166.

29 Paul Ricoeur, entre autres, tout en admettant que certaines critiques du freudisme sont "dévastatrices," s'en prend à tous ceux qui ont entrepris de confronter ce système à la réalité, les accusant de traiter la psychanalyse comme une science d'observation, alors qu'elle serait selon lui une herméneutique et une exégétique. Nous reconnaissons certes volontiers que cet auteur est très bien documenté, très brillant, et très profond, mais nous ne pouvons nous empêcher de nous poser une question: a-t-il le droit de traiter l'oeuvre de Freud comme une oeuvre d'art ou un morceau de poésie, alors que Freud n'a cessé de la présenter comme une 


\section{6 / Renaissance and Reformation}

oeuvre scientifique, reposant sur l'observation et l'expérimentation, comme une oeuvre exprimant la réalité et destinée à la transformer? Voir Paul Ricoeur, De l'interprétation, essai sur Freud (Paris: Seuil, 1965), en partic. pp. 338-366.

30 Inutile de dire que, malgré cela (ou à cause de cela), l'oeuvre de Rabelais présente un in térêt psychologique évident. Glauser écrit, "L'élément pittoresque prédomine sur l'élément psychologique, mais la psychologie est loin d'être absente; elle trouve un corps, elle fait éclater les cadres." Defaux, de son côté, affirme que rien ne lui semble plus erroné "que de refuser à Rabelais des dons comparables à ceux de Molière dans le domaine de la création psychologique." Cette convergence des deux auteurs est remarquable si l'on songe que Glauser est le premier à avoir analysé l'ensemble de l'oeuvre de Rabelais dans l'esprit de la critique moderne, et que Defaux est l'un de ceux qui ont le mieux montré la pertinence et la fécondité de la critique historique. Dans leurs ouvrages magistraux, ces auteurs prouvent, le premier, que le critique peut recréer l'oeuvre d'un écrivain sans nullement la trahir, et le second, qu'il peut explorer la pensée authentique d'un écrivain tout en faisant oeuvre créatrice. Voir:

- Alfred Glauser, Rabelais Créateur (Paris: Nizet, 1966), p. 166.

- Gérard Defaux, Pantagruel et les Sophistes (La Haye: Martinus Nyhoff, 1973), p. 199 et aussi p. XII).

31 Ce qui n'empêche qu'on peut dire, selon l'excellente formule de Larmat, que "le vrai miroir du monde est celui de Rabelais, non celui de Madame de la Fayette." Voir Jean Larmat, Le Moyen Age dans le Gargantua de Rabelais (Paris: Les Belles Lettres, 1973), p. 84. Cet auteur situe très bien dans le temps l'importance que Rabelais accorde à la nourriture, à la scatologie et à la sexualité (pp. 43-86). Dans un autre ouvrage, Larmat écrit que "la critique contemporaine. . .néglige trop aisément ce que Rabelais doit au réel, sans lequel son oeuvre n'existerait pas." Cf Rabelais (Paris: Hatier, 1973), pp. 225-226.

32 A condition toutefois qu'ils puissent surmonter (une bonne psychanalyse y suffira) leur “amnésie infantile." D'après Freud, en effet, nous "oublions" les événements de notre petite enfance, les rejetant dans notre inconscient, pour échapper à l'angoisse de savoir quels pervers (polymorphes) nous avons été. Voir Vocabulaire de la Psychanalyse, p. 22. 